\title{
Comparative Evaluation of Lipid Profile in Ischemic and Hemorrhagic Stroke - A Case Control Study
}

\author{
Gurmeet Singh ${ }^{1}$, Sheikh M.saleem ${ }^{2}$, AB Ahad Wani ${ }^{3}$ \\ ${ }^{1}$ Consultant, Department of Medicine, District Hospital, Baramulla, J \& K, ${ }^{2}$ Additional Professor, Department of Neurology, \\ Sheri Kashmir Institute of Medical Sciences, Soura, Srinagar, J \& K, ${ }^{3}$ Lecturer, Department of Medicine, Skims Medical \\ College, Bemina, J \& K, India
}

Corresponding author: Dr Gurmeet Singh, Consultant, Department of Medicine, District Hospital, Baramulla, J \& K, India

DOI: http://dx.doi.org/10.21276/ijcmsr.2019.4.2.14

How to cite this article: Gurmeet Singh, Sheikh M.saleem, AB Ahad Wani. Comparative evaluation of lipid profile in ischemic and hemorrhagic stroke - a case control study. International Journal of Contemporary Medicine Surgery and Radiology. 2019;4(2):B61-B64.

\section{A B S T R A C T}

Introduction: Stroke is a devastating and disabling cerebrovascular disease with significant amount of residual deficit leading on economic loss. To spread awareness about the common risk factors has decreased the incidence of stroke. Hence, the aim of the present study was to evaluate the lipid profile pattern among ischemic, haemorrhagic strokes and to compare with the control patients admitted to the hospital without any previous history of stroke.

Material and Methods: This study was conducted among patients with diagnosis of stroke comprising 150 patients with 50 each in ischemic, haemorrhagic and in the control group. Clinical diagnosis was established and CT scan of brain without contrast injection was performed to stratify the patient into each category. A detailed history with physical examination was collected from patient's records. Patients with arterial hypertension, diabetes mellitus, and on lipid lowering therapy were not included in the study.

Results: The mean age of patients with stroke was found to be $64 \pm 12$ years with a male to female ratio of 2:1. Out of 150 patients ischemic stroke patients, raised serum total cholesterol was seen in $52 \%$, LDL-cholesterol in $88 \%$ and $\mathrm{HDL}$-cholesterol in $44 \%$ patients whereas in haemorrhagic stroke patients, the serum total cholesterol and triglycerides was raised in $64 \%$ and $28 \%$ patients, LDL-cholesterol in $92 \%$ of the subjects respectively. In control group, there was no difference found in the lipid profile of both the types of stroke.

Conclusion: It was found that haemorrhagic stroke patients had higher serum total cholesterol level i.e. 92\% and lower HDL-cholesterol levels i.e. $24 \%$ as compared to subjects of ischemic stroke. There was no statistical difference in the lipid profile of the subjects with both the types of stroke. Hence, high total and LDL cholesterol are important risk factors in the development of both the types of stroke.

Keywords: lipid profile, Ischemic, Haemorrhage, Stroke, risk factors

\section{INTRODUCTION}

Stroke is defined as rapidly developing symptoms and/or signs of focal and global loss of cerebral function lasting for at least 24 hours with no apparent cause other than of vascular origin. Stroke is the cause of one in eight deaths. It also constitutes a dreadful burden of disability for the patients and their relatives. Stroke describes a clinical event caused either by haemorrhage or occlusion in the blood supply to the central nervous system leading to tissue infarction. ${ }^{1}$

In a study reported by Millikan and Siekert, it was found that worsening of neurological symptoms after a stroke has always awakened interest among clinicians. Attempts to find predictors of neurological worsening showed controversial results. Although vascular disease is the prime contributor to its pathogenesis, dyslipidaemia is not undoubtedly established as a risk factor for stroke in the same way that it is for coronary artery disease. ${ }^{2}$

Evidence of a causal relation between lipid profile and stroke is inconsistent and most large-scale studies on cholesterol and stroke risk have not differentiated between ischemic and haemorrhagic stroke, nor did they differentiate among various subtypes of ischemic stroke. Ischemic is that type of stroke where there is blockage of blood vessels due to which lack of blood flow to the affected area takes place whereas haemorrhagic stroke is the rupture of blood vessels due to which there is leakage found in the affected area. ${ }^{3}$

Due to better preventive measures, the incidence and mortality of stroke has major difference in variety of populations. Risk factors and nonmodifiable factors such as age, gender, familial trends, race and ethnic groups including factors like hypertension, cardiac disease, diabetes mellitus, dyslipidaemia, smoking, alcohol abuse, physical inactivity, asymptomatic carotid stenosis and transient ischemic attacks which have been studied as far. ${ }^{4}$

The relationship of serum lipids and lipoproteins with cerebrovascular disease has been studied with risk factors such as coronary heart diseases. Different types of clinical 
trials depicted an association between high concentrations of serum cholesterol and ischemic stroke. In case-control studies of stroke, cholesterol as a risk factor has usually showed negative findings and prospective studies have failed to show a direct and strong association. .,6 $^{2}$

Globally, with reference to $\mathrm{WHO}$ figures, death rate was found to be 5.8 million in 2005 and is expected to rise to 6.5 million in 2015 and 7.8 million in 2030. It is recognized as a major health burden whether of ischemic or haemorrhagic origin. Worldwide, it is considered to be the second most common cause of death, disability in adults and dementia. ${ }^{7}$ There are modifiable and non-modifiable risk factors for stroke that increase the chances for ischemic stroke. Non modifiable risk factors include old age, ethnicity, male gender, family history and prior history of stroke. Modifiable risk factors can be subdivided into lifestyle and behavioural risk factors and non-lifestyle factors. Modifiable lifestyle risk factors include cigarette smoking and illegal usage of drug. ${ }^{8}$ The non-lifestyle risk factors such as low socioeconomic status, arterial hypertension, dyslipidemia, heart disease and asymptomatic carotid artery disease also increases the probability of ischemic stroke. For patients with blood pressure of $120 / 80 \mathrm{mmHg}$ or higher lifestyle modification as initial therapy is recommended with incorporation of effective lifestyle interventions that includes weight loss, less intake of alcohol, aerobic physical activity, adequate potassium intake, reduction in sodium intake, and dietary regimens as provided by the Dietary Approaches to Stop Hypertension (DASH) eating plan. ${ }^{9}$

It was found from the previous literature that there exists an inverse relation between total cholesterol and death from haemorrhagic stroke. Hence, the association between cholesterol and stroke may not be direct as for coronary heart disease. Different clinical studies have shown that increased serum cholesterol is a risk factor for ischemic stroke due to this reason it is important to evaluate the difference in serum lipid levels in subtypes of strokes to appropriately guide lipid-lowering therapy that can decrease the incidence of stroke and related mortality. ${ }^{10}$ Therefore, the aim of the present study was to assess the lipid profile of patients with ischemic and haemorrhagic stroke and to compare it with the control group.

\section{MATERIAL AND METHODS}

The present study was a case-control study which was conducted at Sher-I-Kashmir institute of Medical science (SKIMS) Kashmir over a period of one 1 year. Total 150 subjects with 20 years to more than 60 years of age were selected with a diagnosis of ischemic (50 subjects) and haemorrhagic strokes (50 subjects) which were compared with controls (50 subjects) in this study. The control subjects were admitted to the hospital for illness other than stroke and do not possess any previous history of stroke. Stroke was defined according to $\mathrm{WHO}$ definition as, rapid onset of a neurological deficit attributed to obstruction or rupture in the cerebral arterial system. Clinical diagnosis was established and CT scan of brain without contrast injection was performed to stratify the patient into each category.

A detailed history with physical examination was collected from patient's records. Patients with arterial hypertension, diabetes mellitus, and on lipid lowering therapy were not included in the study. Also, patients with brain tumour, trauma in head, transient ischemic attack, syncopal attacks and presumptive diagnosis of stroke with no evidence on CT were also excluded in this study. On the next morning after admission, samples of serum were obtained after 12 hours of overnight fasting.

The blood samples were collected into plain tubes after which the samples were centrifuged at $4^{\circ} \mathrm{C}$ for 15 minutes after incubation of 20 minutes for extraction of serum. With the help of enzymatic colorimetric method using chemistry auto-analyser, the sera were analyzed for serum lipid profile that included total cholesterol, triglyceride, LDL-cholesterol and HDL-cholesterol respectively.

\section{STATISTICAL ANALYSIS}

The data was entered into excel sheet and was analysed using SPSS version 21.0. Descriptive analysis was done in the form of frequency and percentage and was presented in the form of tables. Quantitative data was given in the form of mean and standard deviation. Qualitative data were expressed as percentage. Chi-square test was performed as test of significance, with $\mathrm{p}$ value less than 0.05 taken to be significant.

\section{RESULTS}

In the present study, it was found that haemorrhagic stroke was found in $60 \%$ subjects and ischemic stroke in $44 \%$ of the subjects and $38 \%$ in control subjects among age group of more than 60 years. About $40 \%$ of the subjects in control group were found in the age group of 41-60 years followed by $32 \%$ of the subjects in the ischemic group and $28 \%$ in the haemorrhagic group. The least number of subjects were found in the age group of 20-40 years with $12 \%$ in the haemorrhagic group followed by $24 \%$ in the ischemic group with $22 \%$ in the control group (Table no. 1 ).

In the (Table no.2) majority of the male subjects were found in control group i.e. $64 \%$ followed by $60 \%$ in the ischemic group and $52 \%$ in the haemorrhagic group. The majority of the female subjects were seen in haemorrhagic group i.e. $48 \%$ followed by $40 \%$ in ischemic group and $36 \%$ in control group. It was found in the present study that total cholesterol was

\begin{tabular}{|l|c|c|c|}
\hline Age group & Haemorrhagic & Ischemic & Control \\
\hline $20-40$ years & $6(12 \%)$ & $12(24 \%)$ & $11(22 \%)$ \\
\hline $41-60$ years & $14(28 \%)$ & $16(32 \%)$ & $20(40 \%)$ \\
\hline$>60$ years & $30(60 \%)$ & $22(44 \%)$ & $19(38 \%)$ \\
\hline Total & $50(100 \%)$ & $50(100 \%)$ & $50(100 \%)$ \\
\hline
\end{tabular}

Table-1: Shows the distribution of study subjects based on age groups

\begin{tabular}{|l|c|c|c|}
\hline Gender & Haemorrhagic & Ischemic & Control \\
\hline Male & $26(52 \%)$ & $30(60 \%)$ & $32(64 \%)$ \\
\hline Female & $24(48 \%)$ & $20(40 \%)$ & $18(36 \%)$ \\
\hline Total & $50(100 \%)$ & $50(100 \%)$ & $50(100 \%)$ \\
\hline Table-2: Shows the distribution of study subjects based on \\
gender \\
\hline
\end{tabular}




\begin{tabular}{|l|c|c|}
\hline Lipid Profile & Type of Stroke & Mean \pm SD \\
\hline Total Cholesterol & Haemorrhage & $4.72 \pm 1.62$ \\
\cline { 2 - 3 } & Ischemic & $6.04 \pm 1.22$ \\
\cline { 2 - 3 } & Control & $2.94 \pm 0.96$ \\
\hline Triglycerides & Haemorrhage & $2.04 \pm 0.80$ \\
\cline { 2 - 3 } & Ischemic & $3.12 \pm 1.48$ \\
\cline { 2 - 3 } & Control & $3.12 \pm 1.48$ \\
\hline HDL-Cholesterol & Haemorrhage & $2.14 \pm 0.90$ \\
\cline { 2 - 3 } & Ischemic & $1.26 \pm 0.12$ \\
\cline { 2 - 3 } & Control & $1.26 \pm 0.12$ \\
\hline LDL-Cholesterol & Haemorrhage & $6.10 \pm 2.08$ \\
\cline { 2 - 3 } & Ischemic & $6.24 \pm 2.12$ \\
\cline { 2 - 3 } & Control & $5.24 \pm 1.28$ \\
\hline
\end{tabular}

Table-3: Shows the distribution of lipid profile among ischemic, haemorrhagic stroke and control subjects

\begin{tabular}{|l|c|c|c|}
\hline Lipid Parameters & Haemorrhage & Control & p value \\
\hline $\begin{array}{l}\text { Serum Cholesterol } \\
>200 \mathrm{mg} \%\end{array}$ & $32(64 \%)$ & $10(20 \%)$ & 0.001 \\
\hline $\begin{array}{l}\text { LDL-C } \\
>100 \mathrm{mg} \%\end{array}$ & $46(92 \%)$ & $38(76 \%)$ & 0.042 \\
\hline $\begin{array}{l}\text { Triglycerides } \\
>150 \mathrm{mg} \%\end{array}$ & $12(24 \%)$ & $14(28 \%)$ & 0.522 \\
\hline $\begin{array}{l}\text { HDL-C } \\
<40 \mathrm{mg} \%\end{array}$ & $14(28 \%)$ & $34(68 \%)$ & 0.034 \\
\hline \multicolumn{3}{|c|}{ Table-4: Shows the distribution of lipid profile among the } \\
\hline \multicolumn{3}{|l|}{ haemorrhage stroke and control subjects } \\
\hline
\end{tabular}

\begin{tabular}{|l|c|c|c|}
\hline Lipid Parameters & Ischemic & Control & p value \\
\hline $\begin{array}{l}\text { Serum Cholesterol } \\
>200 \mathrm{mg} \%\end{array}$ & $26(52 \%)$ & $10(20 \%)$ & 0.006 \\
\hline $\begin{array}{l}\text { LDL-C } \\
>100 \mathrm{mg} \%\end{array}$ & $44(88 \%)$ & $38(76 \%)$ & 0.036 \\
\hline $\begin{array}{l}\text { Triglycerides } \\
>150 \mathrm{mg} \%\end{array}$ & $18(36 \%)$ & $14(28 \%)$ & 0.342 \\
\hline $\begin{array}{l}\text { HDL-C } \\
<40 \mathrm{mg} \%\end{array}$ & $22(44 \%)$ & $34(68 \%)$ & 0.841 \\
\hline \multicolumn{3}{|l|}{ Table-5: Shows the distribution of lipid profile among the } \\
ischemic stroke and control subjects \\
\hline \multicolumn{4}{|l}{}
\end{tabular}

\begin{tabular}{|l|c|c|c|}
\hline Lipid Parameters & Haemorrhage & Ischemic & p value \\
\hline $\begin{array}{l}\text { Serum Cholesterol } \\
>200 \mathrm{mg} \%\end{array}$ & $32(64 \%)$ & $26(52 \%)$ & 0.452 \\
\hline $\begin{array}{l}\text { LDL-C } \\
>100 \mathrm{mg} \%\end{array}$ & $46(92 \%)$ & $44(88 \%)$ & 0.826 \\
\hline $\begin{array}{l}\text { HDL-C } \\
<40 \mathrm{mg} \%\end{array}$ & $12(24 \%)$ & $18(36 \%)$ & 0.221 \\
\hline $\begin{array}{l}\text { Triglycerides } \\
>150 \mathrm{mg} \%\end{array}$ & $14(28 \%)$ & $22(44 \%)$ & 0.548 \\
\hline $\begin{array}{l}\text { Table-6: Shows the distribution of abnormal percentage of } \\
\text { lipid profile among the ischemic and haemorrhage stroke } \\
\text { subjects }\end{array}$ \\
\hline \multicolumn{3}{|c|}{}
\end{tabular}

increased in ischemic group followed by haemorrhagic group and was less in control group. The triglycerides were also found to be higher among the subjects of ischemic group followed by rise in control group and was least seen in haemorrhagic group. The HDL-C was higher in the subjects of haemorrhagic group and was less among subjects with ischemic and control group whereas the LDL-C was higher in the subjects of ischemic group and was comparatively less in subjects of haemorrhage group followed by further decline in control group (Table no.3).

The lipid parameters showed that when subjects in haemorrhagic group were compared with the control group, the serum cholesterol was higher among haemorrhagic group i.e. $64 \%$ followed by $20 \%$ in the control group which was found to be highly statistically significant at $\mathrm{p}$ value 0.001 . When LDL-C was compared, it was found that it was higher in haemorrhagic group i.e. $92 \%$ followed by $76 \%$ in the control group and was also found to be highly statistically significant at $\mathrm{p}$ value 0.042 . The triglyceride level was higher in control group i.e. $28 \%$ followed by $24 \%$ in the haemorrhagic group which was not found to be statistically significant. The HDL-C was also raised in control subjects i.e. $68 \%$ followed by $28 \%$ in the haemorrhagic group and this was found to statistically significant at $p$ value 0.034 (Table no.4).

When the subjects in ischemic group were compared with the control group, it was observed that serum cholesterol was higher in ischemic group i.e. $52 \%$ and $20 \%$ in the control group which was found to be statistically significant at $p$ value 0.006 . The $\mathrm{LDL}-\mathrm{C}$ was also higher in ischemic group i.e. $88 \%$ followed by $76 \%$ in the control group and this was found statistically significant at $\mathrm{p}$ value 0.036 . The HDL-C was higher in control group i.e. $68 \%$ followed by $44 \%$ in ischemic group. The Triglycerides was more in ischemic group i.e. among $36 \%$ of the subjects followed by $28 \%$ in the control group which was not found to be statistically significant (Table no.5).

It was found that LDL-C was higher among $92 \%$ of the subjects in haemorrhagic group and $88 \%$ of the subjects in ischemic group which was not found to be statistically significant. The least raised parameter was HDL-C which was $24 \%$ in haemorrhagic group and $36 \%$ in the ischemic group and was not significant statistically (Table no.6).

Discussion

There are variety of traditional risk factors which includes smoking, diabetes, hypertension and dyslipidemia for coronary artery disease and stroke. But unlike in coronary artery disease the evidence for the role of these risk factors in stroke is less convincing. Among the risk factor smoking and hypertension are said to be the most important ones. The influence of diabetes on the burden of stroke is more controversial. It includes the spectrum of high LDLcholesterol, high triglyceride and low HDL cholesterol. It is well proven by epidemiological studies that high LDL-C and low HDL-C is associated with cardiovascular disease. Ischemic stroke is basically the result of three known pathogenic mechanisms that includes the involvement of large artery as atherosclerosis in extra-cranial and large intracranial arteries followed by embolism from the heart and intracranial small-vessel disease. Transient ischemic attack is a temporary neurologic deficit caused by a cerebrovascular disease with no clinical or imaging trace. It can be represented as regression of a focal stroke syndrome that reverses completely and dramatically over a period of 
minutes or up to one hour.

In a study done by Togha et al found that the risk of developing ischemic stroke increases as the total cholesterol and LDL values rise. MRFIT study showed an inverse relation between cholesterol level and haemorrhagic stroke, while the risk increased for ischemic stroke with cholesterol levels more than $200 \mathrm{mg} / \mathrm{dl}$, and more than doubled when serum cholesterol values rose above $280 \mathrm{mg} / \mathrm{dl} .{ }^{11,12}$

It was found in the present study that haemorrhagic stroke was found in $60 \%$ subjects and ischemic stroke in $44 \%$ of the subjects and $38 \%$ in control subjects among age group of more than 60 years. The cerebral atherosclerosis with atheroma formation is the basic known pathophysiologic mechanism in ischemic stroke. There are contradictory results from the previous literature regarding correlation between the total plasma cholesterol and the risk of stroke among patients. In an Another study done by Qizilbash et al. it was examined that the relationship between serum total cholesterol and subsequent stroke and found that there was a significant association however, other studies were less conclusive. It was found in the study done by Khan et al that hyperlipidaemia was seen in $16 \%$ patients with stroke and was given as the $3 \mathrm{rd}$ most common risk factor of stroke. But in a study done by Tanveer et al., hyperlipidaemia was found in $21 \%$ of all 200 patients of stroke. ${ }^{13,14,15}$

HDL cholesterol is conventionally considered as the "good cholesterol". But now the seeds of doubt have been sowed-is HDL cholesterol really "good" at all levels. Too much of even good things are not good. Recent studies are of the opinion that values of HDL cholesterol more than $58 \mathrm{mg} \%$ may in fact be more harmful than good. An Iranian study did not find any influence for HDL cholesterol on stroke. ${ }^{16}$

For cerebral infarction, diabetes mellitus has been regarded as prominent risk factor. It contributes to atherosclerosis of the cerebral arteries that can affect the cerebral blood flow leading to both small-vessel and large vessel stroke and lacunar infarction. To increase HDL cholesterol levels therapeutic intervention such as modifications in lifestyle, for example; increased exercise, smoking cessation, moderate alcohol consumption and adoption of a mediterranean diet is required. ${ }^{17}$

\section{CONCLUSION}

There was no statistical difference observed in the lipid profile with the two types of stroke. It was found that haemorrhagic stroke patients were having higher serum total cholesterol and higher HDL-cholesterol levels when compared with subjects of ischemic stroke. Hence, high risk patients with stroke should be screened using serum lipid profile and further studies with larger sample size are required to find out the outcome of lipid lowering therapy related to morbidity and mortality among ischemic stroke patients.

\section{REFERENCES}

1. Ansari AK, Akhund IA, Shaikh AQ. Stroke in elderly: Identification of risk factors. J Ayub Med Coll Abottabad 2001; 13(1): 11-13.

2. Austin MA, King MC, Vranizan KM, Krauss RM. Atherogenic lipoprotein phenotype. A proposed genetic marker for coronary heart disease risk. Circulation. 1990; 82 (4):495-506.

3. Musunuru K. Atherogenic Dyslipidemia: Cardiovascular Risk and Dietary Intervention Lipids. 2010; 45(10):907-914.

4. Togha M, Gheini MR, Ahmadi B, Razeghi PKS. Lipid profile in cerebrovascular accidents Iran J Neurol. 2011; 10(1-2):1-4

5. Lindenstrom E, Boysen G, Nyboe J. Influence of total cholesterol, high-density lipoprotein cholesterol, and triglyceride on risk of cerebrovascular disease. BMJ1994; 309 (1):11-15.

6. World Health Organization. The world health report: shaping the future. Geneva: WHO; 2003. Last accessed on $12^{\text {th }}$ January 2019.

7. Khan NI, Naz L, Mushtaq S, Rukh L, Ali S, Hussain Z. Ischemic stroke: prevalence of modifiable risk factors in male and female patients in Pakistan. Pak J Pharm Sci 2009; 22 (6):62-67.

8. Pandian JD, Sudhan P. Stroke epidemiology and stroke care services in India. Journal of Stroke. 2013; 15 (4):128-34.

9. Wasay M, Khatri IA, Kaul S. Stroke in South Asian countries. Nat Rev Neurol. 2014; 10 (2):135-43.

10. Austin MA, King MC, Vranizan KM, Krauss RM. Atherogenic lipoprotein phenotype. A proposed genetic marker for coronary heart disease risk. Circulation. 1990;82 (5):495-506.

11. Togha M, Gheini MR, Ahmadi B, Khashaiar P, Razeghi S. Razeghi S. Lipid profile in cerebrovascular accidents. Iran J Neurol. 2011; 10(1-2):1-4.

12. Iso H, Jacobs DR, Wentworth D, Neaton JD, Cohen JD. For the MRFIT Research Group. Serum cholesterol levels and six-year mortality from stroke in 350,977 men screened for the multiple risk factor intervention trial. N Engl J Med. 1989; 320 (3):904-910.

13. Qizilbash N, Duffy SW, Warlow C, Mann J. Lipids are risk factors for ischemic stroke: overview and review. Cerebrovasc Dis 1992; 2 (1):127-136.

14. Khan H, Afridi AK, Saadia A. A hospital based study on stratification of risk factors of stroke in Peshawar. Pak J Med Sci 2006; 22 (5):304-307.

15. Tanveer A. Localization and management in Cerebrovascular accident: a comparison of clinical assessment versus C.T scans [Dissertation]. College of Physicians \& Surgeons Pakistan; Karachi: 1996.

16. Mary Grace, K. J. Jacob, Arya Vijay Kumar, Shameer V. K. Role of dyslipidemia in stroke and comparison of lipid profile in ischemic and hemorrhagic stroke -a case control study. Int J Adv Med. 2016;3(3):694-698.

17. Appel LJ, Moore TJ, Obarzanek E, Vollmer WM, Svetkey LP, Sacks FM, Bray GA, Vogt TM, Cutler JA, Windhauser MM, Lin PH, Karanja N. A clinical trial of the effects of dietary patterns on blood pressure. DASH Collaborative Research Group. N Engl J Med 1997; 336(16):1117- 1124.

\section{Source of Support: Nil; Conflict of Interest: None}

Submitted: 30-10-2018; Accepted: 10-11-2018; Published online: 22-05-2019 